\title{
Energy Efficient Routing Strategies for Clustered Wireless Sensor Networks: An Analytical Framework
}

\author{
S. Jeba Anandh \\ Research Scholar \\ Anna University, Chennai \\ India
}

\author{
E. Baburaj \\ Professor, Dept. of CSE \\ Sun College of Engg. And Tech. \\ Tamil Nadu, India
}

\begin{abstract}
The efficiency of a wireless sensor network depends on its life time. By conserving the battery power of individual sensor devices, considerable amount of total energy can be saved thereby increasing the total lifetime of the network. The operation phases of a wireless sensor network comprises of sensing the data, aggregating the data in an intermediate node, communicating and forwarding the aggregated data to the sink for further process. Among these, communication or routing process requires more energy than any other operation. Hence a better approach would be allowing only a selected number of nodes to communicate with the sink. This idea led to the construction of the sensor network as a number of clusters with a dynamically elected cluster-head node is only allowed to forward the data to the sink. Various routing techniques for clustered Wireless Sensor Networks has emerged yielding good result in improving the lifetime of the network. Here in this paper, a survey of these routing techniques are presented.
\end{abstract}

\section{Keywords}

Clustering, Cluster Head, Data Aggregation, Routing Protocols

\section{INTRODUCTION}

A typical WSN consists of a large number of low-cost, low-power, and multifunctional wireless sensor nodes, with sensing, wireless communication and computation capabilities. These sensor nodes communicate over short distance using a wireless medium and collaborate to perform common tasks such as environmental monitoring, military surveillance, industrial process control and habitat monitoring[1]. On deployment, the battery powered sensor nodes must be able to autonomously organize themselves into a wireless communication network. As the sensor nodes operate unattended for the remaining period of their life, it is impractical to recharge them. This requires the development of new protocols to efficiently utilize the power availability in sensor nodes. Routing[2] in sensor networks provide a number of challenges. First, it is difficult to build a global addressing scheme for the deployment of large number of sensor nodes. Hence, classical IP-based protocols cannot be incorporated to the sensor networks. Second, all data sensed by individual data should be forwarded to sink or base station. Third, the data traffic generated by the network may contain redundancy in it because multiple sensors may be generating same data within the vicinity of a phenomenon.
To overcome these shortcomings and to support data aggregation through efficient network organization, the sensor nodes can be categorized into a number of small groups called clusters. Each cluster has a coordinator, referred to as a cluster head, and a number of member nodes. Clustering[3] is a

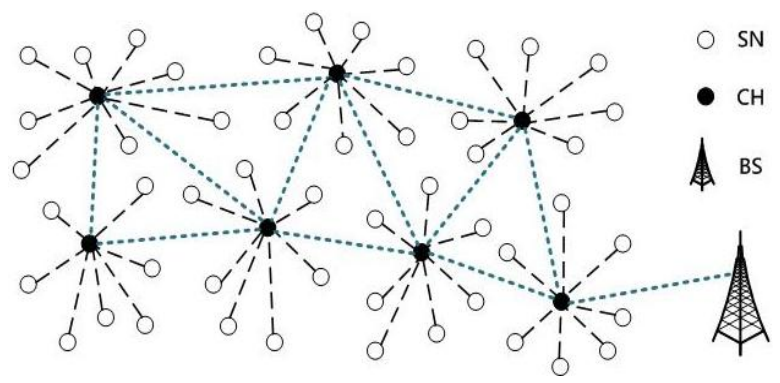

Figure. 1 Operation of a clustered wireless sensor network hierarchical architecture in which Cluster Heads[4] (CHs) form the higher level while member nodes form the lower level. The member nodes report their data to the respective $\mathrm{CHs}$. The $\mathrm{CHs}$ aggregate the data and send them to the central base station through other $\mathrm{CHs}$. Because $\mathrm{CHs}$ often transmit data over longer distances, they lose more energy compared to member nodes. The network may be reorganized periodically in order to elect energy-rich nodes to serve as $\mathrm{CHs}$, thus distributing the load uniformly to all the nodes. Besides achieving energy efficiency, clustering reduces channel contention and packet collisions, resulting in better network throughput under high load. Clustering has been shown to improve network lifetime, a primary metric for evaluating the performance of a sensor network. The Figure 1 shows the operation of clustering in WSN.

\section{ADVANTAGES OF CLUSTERING IN WSN}

This section enumerates the various advantages of using clustering architecture in WSN. Some of them are

\subsection{Scalability:}

In clustering routing scheme, sensor nodes are categorized into a number of clusters with different sizes. The Cluster Heads of each cluster are responsible for data aggregation, information dissemination and network management. Localized route management in intra-clusters reduces the routing table size stored at individual sensor nodes. This enables newer sensor nodes to be included dynamically and thus ensures scalability. 


\subsection{Data Aggregation:}

Data aggregation from multiple nodes by the cluster head avoids redundant forwarding of data to the sink node. By avoiding individual non cluster head nodes to aggregate data, large amount of energy can be saved.

\subsection{Load distribution:}

Since most of the processing is done by the cluster heads and other nodes performing only data forwarding operation, careful selection of cluster heads would ensure the load to be distributed evenly to all the nodes of the network.

\subsection{Energy Consumption:}

Data aggregation by cluster heads reduction of long distance communication due to intra and inter cluster communications and other factors contribute to the conservation of a large amount of energy.

\subsection{Robustness:}

Clustering routing scheme makes it more convenient for network topology control and responding to network changes comprising node increasing, node mobility and unpredicted failures, etc. A clustering routing scheme only needs to cope with these changes within individual clusters, thus the entire network is more robust and more convenient for management. In order to share the $\mathrm{CH}$ responsibility, $\mathrm{CHs}$ are generally rotated among all the sensor nodes to avoid the single point of failure in clustering routing algorithms.

\subsection{Collision Avoidance:}

In a clustered architecture, due to the possibility of intra and inter cluster communication, transmission takes place in a controlled fashion, so that possibility of data collision is minimal.

\subsection{Fault-Tolerance:}

Due to the enormous deployment of sensor nodes at adverse environments, sensor nodes may suffer from energy depletion, transmission errors, hardware malfunction and security risks. These fault conditions if occur can easily be localized and rectified in a clustered environment. Reclaiming the data from faulty sensor node is quite easy as cluster heads of these nodes periodically collects data from its member nodes.

\subsection{Ease of Connectivity:}

Due to the mobility of sensor nodes, frequent disconnection of nodes from their clusters happen. Re-association of the disconnected nodes is quite easy with the issue of only a fewer control signal. When newer nodes are being deployed, their association takes place with the nearest cluster heads identified using signal strength.

\subsection{Energy Hole Avoidance:}

Nodes nearer to sink may need to forward more data resulting in energy depletion at these nodes. This energy-hole problem can be avoided by constructing relatively smaller clusters near sink node so that intra communication in these smaller clusters would be minimal.

\subsection{Network Lifetime:}

Network lifetime[5] is influenced by power supply, processing capability and bandwidth. In order to prolong network life time, energy rich nodes should be chosen as cluster head and route select selection towards sink should include energy rich nodes.

\subsection{Quality of Service:}

The complex applications and the functionalities of WSNs prompt the necessity of quality of service (QoS). High throughput, less delay and good utilization of bandwidth are required to maintain quality of service. It is difficult for all the routing protocols to satisfy these requirements of QoS, because some demands may breach one or more protocol principles. Mostly clustering routing approaches in WSN mainly focuses on increasing energy efficient rather than QoS provisioning.

\section{NETWORK DESIGN CHALLENGES AND ROUTING ISSUES}

The design[6] of routing protocols for WSNs is highly challenging as several network constraints such as energy, bandwidth, processing unit, and storage pose greater hurdles. Some of the design challenges encountered in routing in sensor networks are listed in this section.

\subsection{Limited energy Availability:}

Since wireless sensor nodes are powered with small sized batteries, they have limited energy capacity[7] available for operation. As WSN are employed in adverse environmental conditions, it is mostly not possible to recharge the batteries of these nodes. Example adverse environments are battle fields, forest region etc. Furthermore, when the energy of a sensor reaches a certain threshold, the sensor will become faulty and will malfunction, which will have a major impact on the network performance. Thus, routing protocols designed for sensors should be as energy efficient as possible to extend their lifetime, and hence prolong the network lifetime while guaranteeing high all-round performance.

\subsection{Locating Sensors:}

Once deployed, the sensor nodes move around autonomously over a sensing region to gather data of interest. To find the locations[8] of sensor nodes, they must be employed with GPS service or any localization techniques. But these strategies too may backfire in hostile environmental conditions leading to loss of node consequently the data it holds.

\subsection{Hardware resource Constraints:}

Due to their small size, sensor nodes have only limited processing and storage capacities, and thus they can only perform limited computations. This will have direct implications on routing design, as routing may need lot of computations to find a new route and store routing tables at intermediate nodes.

\subsection{Application oriented deployment:}

In Wireless Sensor Networks, node deployment is mostly dependent on the application on which it is deployed. In such scenarios, the nodes are randomly deployed over an area of interest that may be hostile in nature. Incorporating routing protocols over these kinds of inaccessible regions may result in high overhead in collecting data towards the base station. Even clustering becomes difficult as nodes get scattered in such an uneven node distribution.

\subsection{Dynamic Network Topology:}

Usually wireless sensor networks are deployed in dynamic and unreliable environmental conditions. The topology[9] of the network is defined by the mobility of sensor nodes and hence the network links between the sensor nodes, changes often due to addition of nodes, nodes going out-of-range, node failures or damages caused by energy depletion or hardware 
malfunctioning. Due adverse deployment conditions, the wireless medium connecting the nodes are prone to high noise and data loss. Hence energy efficient routes should be the ultimate goal in the design of the routing protocol. By regularizing the mobility pattern of the nodes, localizing the faulty nodes, defining new dynamic paths in case of route breaks would lead to achieve the routing goal.

\subsection{Data Aggregation:}

Sensor networks are known to generate redundant data from multiple sources. Proper data aggregation technique should identify and categorize these data, so that the data transmission itself would aid energy harvesting. Importantly Data-centric routing protocols should utilize efficient data aggregation techniques in forwarding the data.

\subsection{Cluster Size:}

In clustered type of architecture, equal sized clusters or uneven clusters may result in data loss, as there is a possibility of black-hole generation near the base station. Larger clusters near the BS would mean higher intra-cluster energy consumption, leading to this $\mathrm{CH}$ getting depleted so quickly that the entire data forwarded to this $\mathrm{CH}$ may not be reaching the BS. So cluster organization itself aids in efficient routing.

\subsection{Scalability:}

Routing protocols design should be such that it should scale along with the network size. Routing protocols should consider scaling sensor nodes having different capabilities in terms of energy, processing, sensing, and transmission range. Hence, as communication links are asymmetric in nature, routing protocols should support efficient data transmission through these kinds of links.

\section{CLUSTERING ROUTING PROTOCOLS IN WSN}

In this section, a detailed survey on important clustering routing protocols for WSNs is presented. Furthermore, this section analyzes the operation of these protocols, and highlights their characteristics with advantages and disadvantages.

\subsection{LEACH:}

Low-Energy Adaptive Clustering Hierarchy (LEACH), proposed by Heinzelman et al. [10], is one of the important clustering routing approaches for WSNs. The basic idea of LEACH was used to develop many other clustering routing protocols. The main objective of LEACH is to elect sensor nodes as $\mathrm{CHs}$ by rotation, so the high energy dissipation in communicating with the BS is spread to all sensor nodes in the network.

The operation phases of LEACH are separated into two phases, the set-up phase and the steady-state phase. In the setup phase the clusters are organized and data is sensed, whereas in the steady-state phase data is forwarded to the base station. During the set-up phase, each node makes a decision of whether to become a $\mathrm{CH}$ for that round. This decision is based on the suggested percentage of $\mathrm{CHs}$ for the network and the number of times the node has been a $\mathrm{CH}$ so far. This decision is made by the node choosing a random number between 0 and 1 . The node becomes a $\mathrm{CH}$ for the current round if the number is less than the following threshold:

$$
T(n)=\left\{\begin{array}{l}
P \\
\frac{P}{1-P\left(\bmod \frac{1}{P}\right)}, \forall \mathbf{n} \in \mathrm{G} \quad \ldots . . .(1)
\end{array}\right.
$$

where $\mathrm{P}$ is the desired percentage of $\mathrm{CHs}, \mathrm{r}$ is the current round, and $\mathrm{G}$ is the set of nodes that have not been elected $\mathrm{CHs}$ in the last $1 / \mathrm{P}$ rounds. When a node gets elected as $\mathrm{CH}$, it informs this message to all other nodes. According to the received signal strength of this control signal, other nodes decide to which cluster head to join for the current round and send an association request to that $\mathrm{CH}$. In order to evenly distribute energy load among sensor nodes, $\mathrm{CHs}$ rotation is performed at each round by generating a new advertisement phase. During the steady-state phase, the sensor nodes sense and transmit data to the $\mathrm{CHs}$. The $\mathrm{CHs}$ compress the data received from member nodes of the cluster, and send an aggregated or fused data packet to the BS directly. Besides, LEACH uses a TDMA/CDMA MAC to reduce inter-cluster and intra-cluster collisions. After a random period of time, the network returns back into the set-up phase again and performs another round of $\mathrm{CH}$ election. Then again steady state phase is continued until all rounds of operation are performed. The different phases of operation can be realized using the fig. 2 .

The various advantages of using LEACH include a node that served as a $\mathrm{CH}$ in a particular round cannot be selected as the $\mathrm{CH}$ again, ensuring all nodes can equally share the load. Another advantage is providing a TDMA schedule to the $\mathrm{CH}$ prevents collisions with other nodes. Lastly, Cluster members avoid excess energy dissipation by using fixed time slots.

There are some disadvantages too in LEACH. They are, single hop inter cluster communication to the base station is not suitable for long range communication. Another disadvantage is $\mathrm{CHs}$ rotation is performed at each round to achieve load balancing, LEACH cannot ensure real load balancing in the case of sensor with different amounts of initial energy, because $\mathrm{CHs}$ nodes are elected in terms of probabilities without energy considerations. Sensor nodes, with lower initial energy, that act as $\mathrm{CHs}$ for the same number of rounds as other sensor nodes, with higher initial energy, will die prematurely. This could bring about energy holes[11] and coverage problems. Next, due to the $\mathrm{CH}$ election is performed in terms of probabilities, it is not guaranteed for the $\mathrm{CHs}$ to be uniformly distributed throughout the network. Therefore the possibility is that the elected $\mathrm{CHs}$ may get deployed in the same area and some nodes may not be able to access any of the $\mathrm{CHs}$ at all. Lastly, dynamic cluster construction needs more control signals to be circulated during every setup phase which aides increased energy consumption. 


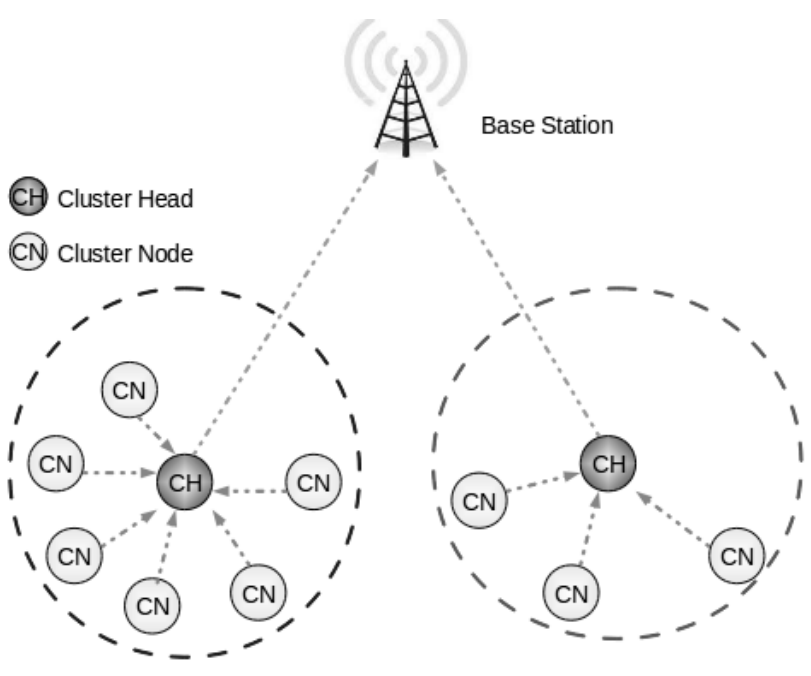

Figure. 2 Working principle of LEACH protocol

\subsection{HEED:}

Hybrid Energy-Efficient Distributed clustering (HEED) [12], was introduced by Younis and Fahmy. This is a multi-hop WSN clustering algorithm which brings an energy-efficient clustering routing that gives importance to energy conservation. In contrast with the cluster head election in LEACH, HEED does not select CHs randomly. HEED uses two parameters in constructing clusters. One parameter depends on the node's residual energy, and the other parameter is the intra-cluster communication cost. In HEED, elected $\mathrm{CHs}$ have relatively higher average residual energy compared to member nodes. Additionally, one of the main goals of HEED is to get evenly-distributed $\mathrm{CHs}$ throughout the network. The probability of two nodes within each other's communication range becoming cluster heads is minimal in HEED. Initially Cprob, percentage of CHs among all nodes, , is set to an optimal value. The probability that a node becomes a $\mathrm{CH}$ is:

$$
C H_{\text {prob }}=C_{\text {prob }} \frac{E_{\text {residual }}}{E_{\max }} \ldots \ldots \ldots .(2)
$$

where Eresidual is the estimated current energy of the node, and Emax is a reference maximum energy, which is typically identical for all nodes in the network. The value of CHprob, however, is not allowed to fall below a certain threshold that is selected to be inversely proportional to Emax. Afterwards, each node is allowed to iterate several times until it finds the $\mathrm{CH}$. If the node is not in $\mathrm{CH}$ list, the node elects itself to be a $\mathrm{CH}$ and sends an announcement message to its neighbors. Each node doubles its CHprob value and goes to the next iteration until its $\mathrm{CHprob}$ reaches 1 . Therefore, there are two types of status that a sensor node could announce to its neighbors: tentative status and final status. If its $\mathrm{CHprob}$ is less than 1 , the node becomes a tentative $\mathrm{CH}$ and can change its status to a regular node at a later iteration if it finds a lower cost $\mathrm{CH}$. If it's CHprob has reached 1, the node permanently becomes a $\mathrm{CH}$. In HEED, every node elects the least communication cost $\mathrm{CH}$ in order to join it. On the other hand, CHs send the aggregated data to the BS in a multi-hop[13] fashion rather than single-hop fashion of LEACH.

The advantages of the HEED protocol are as follows: (1) It is a fully distributed clustering method that benefits from the use of the two important parameters for $\mathrm{CH}$ election; (2) Low power levels of clusters promote an increase in spatial reuse while high power levels of clusters are required for intercluster communication. This provides uniform $\mathrm{CH}$ distribution across the network and load balancing; (3) Communications in a multi-hop fashion between $\mathrm{CHs}$ and the BS promote more energy conservation and scalability in contrast with the single-hop fashion, i.e., long-range communications directly from $\mathrm{CHs}$ to the sink, in the LEACH protocol.

The main disadvantages in HEED are, the possibility of tentative cluster heads not becoming regular cluster heads may leave some uncovered nodes. These nodes may be forced to become a $\mathrm{CH}$ and these forced $\mathrm{CHs}$ may be in range of other CHs or may not have any single member associated with them. As a result, more $\mathrm{CHs}$ are generated than the expected number and this also accounts for unbalanced energy consumption in the network. As with LEACH, the clustering process in each round imposes significant control overhead in the network. This overhead causes noticeable energy dissipation which results in decreasing the network lifetime. Since HEED requires several iterations to form clusters, packet overhead may also be a reality.

\subsection{TL-LEACH:}

Two-Level Hierarchy LEACH (TL-LEACH), [14], is an extension of LEACH. TL-LEACH uses the following two techniques to achieve energy and latency efficiency: randomized, adaptive, self-configuring cluster formation and localized control for data transfers. In TL-LEACH, a $\mathrm{CH}$ collects data from member nodes as LEACH, but instead of transmitting data to the BS directly, it uses other $\mathrm{CHs}$ as relays to forward data to $\mathrm{BS}$.

The algorithm is consists of four basic phases: advertisement phase, cluster setup phase, schedule creation and data transmission. In the initial phase of operation, each node decides whether to become a primary $\mathrm{CH}$ or secondary or remain as ordinary node. If a node is elected as primary $\mathrm{CH}$, it uses CSMA technique to advertise other nodes. Next, secondary $\mathrm{CH}$ nodes send the advertisement to the ordinary nodes. In this phase, each secondary $\mathrm{CH}$ decides on to which primary $\mathrm{CH}$ it should get associated and sends an advertisement message to its primary $\mathrm{CH}$. In the same way, each ordinary node decides on to which secondary $\mathrm{CH}$ it should get associated. In the third phase, each primary $\mathrm{CH}$ creates a TDMA schedule assigning each node in its group a slot to transmit. The primary $\mathrm{CH}$ selects a CDMA code and broadcasts all its member nodes at second level to use this code. Similarly, all secondary $\mathrm{CH}$ can transmit this information to ordinary nodes in its group using both the code and the schedule from the primary $\mathrm{CH}$. In the last phase, clusters are created and each node can transmit with respect to the TDMA[15] schedule decided by its primary $\mathrm{CH}$.

The advantages of TL-LEACH are as follows: (1) TLLEACH uses random rotation of local cluster BSs, i.e., primary $\mathrm{CHs}$ and secondary $\mathrm{CHs}$, which can bring about better energy load distribution across the network; (2) TLLEACH uses localized coordination, which is conductive to scalability and robustness in the network; (3) Compared with $\mathrm{LEACH}$, the scheme of two-levels clustering leads to less average transmission distance, and less nodes are required to transmit far distances to the BS via TL-LEACH. This effectively reduces the total energy consumption.

However, there exist a few disadvantages of TL-LEACH as follows: (1) Despite that the average transmission distance is decreased in comparison with LEACH, the two-hop inter- 
cluster routing of TL-LEACH is still not applicable to largerange networks, because it uses only two hops for data transmission from sources to the BS, and long-distance communications can breed much energy consumption; (2) $\mathrm{CH}$ election without energy considerations assumes an ideal homogeneous network and cannot ensure real load-balancing in case of nodes with different amount of initial energy.

\subsection{PEGASIS:}

Power-Efficient Gathering in Sensor Information Systems[16] (PEGASIS), proposed by Lindsey et al., is an improvement of LEACH. The main idea behind PEGASIS is that every node communicates only with their close neighboring nodes and takes turns to become the leader to transmit to the sink. In PEGASIS, the locations of nodes are random, and each sensor node has the ability of data detection, wireless communication, data fusion and positioning. Initially Energy is evenly distributed among the sensor nodes in the network.

In PEGASIS, the sink organizes the nodes to form a chain or otherwise formed by the nodes themselves using a greedy algorithm. In the latter case, a node can first get the location data of all nodes and locally compute the chain using the same greedy. During the process of chain formation in PEGASIS, all the nodes are assumed to have a global knowledge of the network and the greedy algorithm is employed on them. The chain construction starts from the farthest node from the sink and the closest neighbor to this node will be the next node on the chain. When a node in this chain becomes inaccessible, the chain will be reconstructed in the same manner to bypass that node.

This technique guarantees, in each round, every node receives data from one of its neighbor, fuses the data with its own, and transmits to the other neighbor on the chain. Data movement takes place in this manner and eventually, the fused data reaches the sink through the leader at a random position on the chain. The leader is important for nodes to die at random locations, in respect that the idea of nodes dying at random places is to enhance the robustness of the network. In all rounds, a control token may be passed by the leader to start the data transmission from the farther end of the chain. The following diagram at the end of this section shows the data transmission process in PEGASIS. Fig.3 explains the process of data transmission using PEGASIS.

Some of the advantages of using PEGASIS are it outperforms LEACH for different network sizes and topologies, because it reduces the overhead of dynamic cluster formation in $\mathrm{LEACH}$, and decreases the number of data transmissions through the chain of data aggregation. The energy load is evenly distributed in the network. Leaders are selected in each round according to the current network topology.

There are some disadvantages too in PEGASIS, they are, nodes use multi-hop communications with the sink thereby allowing too much energy consumption. Due to single chain being used to communicate to sink from distant node, there exists an unnecessary delay in the transmission.

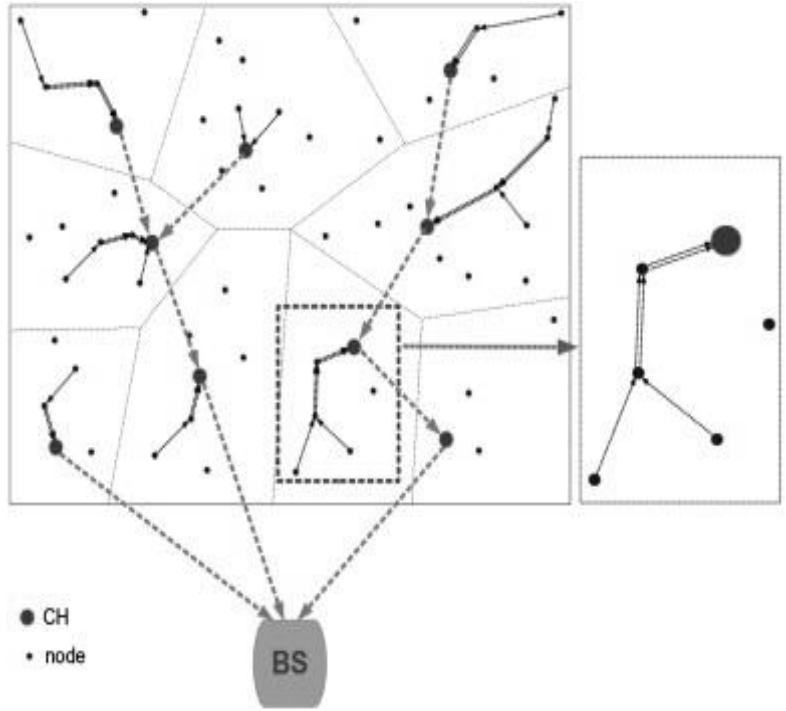

Figure 3 Data Transmission using PEGASIS

\subsection{TEEN:}

This protocol called Threshold sensitive Energy Efficient sensor Network protocol (TEEN) [17], was proposed by Manjeshwar and Agrawal. The main objective of this protocol is to cope with sudden changes in the sensed data. The protocol combines the hierarchical[18] approach with a data-centric approach. As in other applications, the nodes sense their environment continuously, but the energy consumption is usually much less compared with other applications, because in this protocol data transmission is done less frequently.

There are two threshold values defined, hard threshold and soft threshold. The hard threshold is the value for the sensed phenomena and it is the absolute value of the attribute beyond which, the node sensing this value will have to enable its transmitter and forward its data to the cluster head, Whereas, the soft threshold is a small change in the value of the sensed phenomena which triggers the sensing node to switch on its transmitter to transmit. In this protocol, the cluster heads send its members both a hard threshold as well as a soft threshold. Therefore by using this protocol, the hard threshold tries to minimize the data transmissions by allowing the nodes to transmit only when the sensed attribute is in the specified range. Then the soft threshold further minimizes data transmissions by stopping communications when there is little or no change in the sensed phenomena. These threshold values can be changed during cluster change period for appropriate results.

The advantages of TEEN protocol include, using the two threshold values, data transmission can be controlled tremendously that is allowing only the sensitive data to get transmitted. This reduces the energy incurred by frequent transmission. 
Some disadvantages of TEEN are it may not suit for certain applications which require periodic appraising, because the process may not get any data at all if the values of the sensed phenomena may not enter the threshold values. As data transmission depends on the threshold value, the sink node finds it difficult to differentiate active and passive nodes. During the time the value of sensed phenomena matches with the threshold, the cluster heads should be in range so that data may not get lost.

\subsection{APTEEN}

The Adaptive Threshold sensitive Energy Efficient sensor Network protocol (APTEEN) [19] is an extension to TEEN. Its main objective is to allow transmitting periodic data and responding to events fired by thresholds. This protocol dictates the periodic transmission and threshold values used in TEEN according to the application being used. The APTEEN protocol issues different types of queries such as historical, on-time, and persistent.

APTEEN starts with the cluster heads broadcasting four types of parameters to member nodes: (1) Attributes (A): a set of physical parameters which the user is interested in obtaining data about; (2) Thresholds[20]: this parameter consists of the hard threshold (HT) and soft threshold (ST). The hard threshold is the value for the sensed phenomena and it is the absolute value of the attribute beyond which, the node sensing this value will have to enable its transmitter and forward its data to the cluster head. Whereas, the soft threshold is a small change in the value of the sensed phenomena which triggers the sensing node to switch on its transmitter to transmit; (3) Schedule: this is a TDMA schedule, that assigns a time slot to each node; (4) Count time (CT): it is the maximum time period between two successive reports sent by a particular node. It can be a multiple of the TDMA schedule length.

The distinctive approach of APTEEN from other protocol is its ability to switch between proactive and reactive modes to transmit data. As with other protocols, all nodes sense the environment continuously, but only those nodes which sense a data value at or beyond the hard threshold value are only permitted to transmit. If a node does not send data for a time period equal to the count time, it must transmit the data again in a different time. Then all the cluster heads aggregates the data from the member nodes and transmits the aggregated data to the sink. Moreover, APTEEN provides a lot of flexibility by allowing the user to set the CT interval value and thereby the user can control the threshold values for energy consumption by changing the CT.

Certain advantages of APTEEN include APTEEN works in combination of both proactive techniques and reactive techniques. It provides high flexibility in changing the counttime interval value and the threshold values for the energy consumption.

The two main disadvantages of APTEEN need additional parameters to implement the threshold computations and the count time. APTEEN provides additional overhead in cluster construction in multiple levels and implementing threshold functions.

\subsection{MBC}

Mobility based clustering [21] addresses the problem of inefficient cluster head election by electing a node based on its residual energy and its mobility. During the cluster process, an assumed non cluster head node considers its connection time with distance from a cluster head, residual energy and node degree of the cluster head. This provides a stable link with cluster head and thus increases packet delivery rate. $\mathrm{MBC}$ reduces energy consumption by reducing the control overhead.

MBC proceeds as like LEACH with setup and steady state and steady state phases electing the cluster heads. In MBC it additionally considers the speed of each sensor device in the computation for $\mathrm{CH}$. Here the threshold $\mathrm{T}(\mathrm{n})$ of LEACH is computed as

$T(n)=\left\{\begin{array}{l}\frac{P}{1-P\left(r \bmod \frac{1}{P}\right)} \times\left(\frac{\mathrm{E}_{\mathrm{n} \_ \text {current }}}{\mathrm{E}_{\max }} \frac{V_{\max }-V_{n_{-} \text {current }}}{V_{\max }}\right), \forall \mathrm{n} \in \mathrm{G} \ldots \ldots \ldots \text { (3) } \\ 0\end{array}\right.$

where En_current is the current energy, Vn_current is the current speed, Emax is the initial energy and Vmax is the maximum speed of the node. This equation makes sure that $\mathrm{T}(\mathrm{n})$ doesn't grow larger than 1.

With the above process, a cluster head is selected and the $\mathrm{CH}$ broadcasts an advertisement message with attributes such as its location, velocity of the sensor node. Then the individual nodes decide on which to which cluster head to join as in LEACH.

\subsection{BSCDCP}

The Base-Station Controlled Dynamic Clustering Protocol forms clusters based on the fact that the clusters should have balanced energy levels [22]. Here in BSCDCP the base station is chosen to be a node with a large amount of energy supply as it coordinates all other actions in the network. The protocol proceeds with the base station receiving information such as the current energy status from all the nodes in the network. With this information, the base station computes the average energy level of all the nodes. Then the base station selects a number of nodes whose energy levels are higher than the average energy value. These nodes will be designated as cluster heads to which other member nodes will be enacted. Each of the cluster heads will be served with an equal number of member nodes so that network load is uniformly distributed to all the cluster heads. BSCDCP also ensures a uniform placement of cluster heads throughout network.

\subsection{TTDD}

The Two-Tier Data Dissemination protocol assumes that the sensor nodes are stationary whereas the sinks can change their location dynamically [23]. The sensor nodes are fed with the location information of their own in order to appraise the nearby sink. When an event is sensed by the sensor nodes, one of them initiates data processing by creating data reports. This node also initiates the construction of a virtual grid structure in which it acts as the start node in the grid to begin data transmission. It sends a data announcement message to its four different adjacent crossing points using greedy geographical forwarding. This message transmission stops only when either it reaches to a node that is closest to the crossing point or it reaches the boundary of the network. 


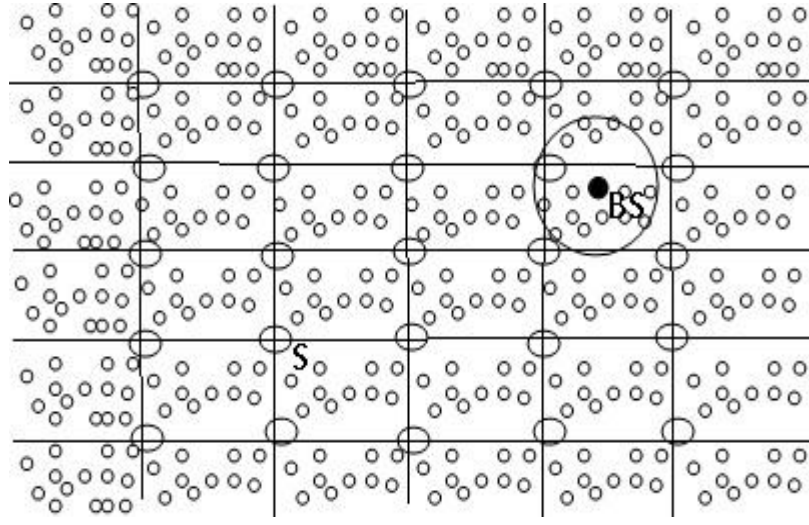

Figure. 4. Grid view of network in TTDD

In figure 4, an example is depicted for the construction of the grid initiated by source node $\mathrm{S}$. In this two dimensional sensor field the source $\mathrm{S}$ divides the field into a grid of cells or clusters when it senses data. This source itself is at one crossing point of the grid. It propagates data announcements to reach all other crossings which also reach base station.

The TTDD can also be used for multiple mobile sinks in a field of stationary sensor nodes. The main limitation here is that each source node may build its own virtual grid structure of dissemination points to supply data to mobile sinks that may lead to more complexity.

\subsection{UCS}

Unequal Clustering Size (UCS) model [24] was proposed by Soro and Heinzelman by organizing the network such that it balances the energy consumption of $\mathrm{CHs}$, thereby increasing the network lifetime. UCS assumes that the $\mathrm{CHs}$ positions are determined with all $\mathrm{CHs}$ arranged inside concentric circles around the BS which is located at the center of the network.

Concentric circles formed can be termed as layers that are bisected by required number of perpendicular numbers thereby creating a known number of clusters. As the circles grow in numbers outward from the BS, the size of the cluster also grows. Fig.5 depicts UCS organization.

The positioning the $\mathrm{CH}$ within the cluster boundaries is very important as it determines the energy consumption by the nodes of that cluster. Keeping the $\mathrm{CH}$ at the centre of the cluster would considerably decrease the total energy consumption within the cluster. By increasing or decreasing the radius of outward concentric circles it is possible to vary the size of the cluster as well the number of nodes contained in a cluster. Data transmission is done through multiple hops, where every $\mathrm{CH}$ chooses to forward its data to the closest $\mathrm{CH}$ in the direction of the BS. The advantages of UCS are: (1) As the number of nodes in a cluster can controlled, it is quite easy to uniformly distribute load among the cluster heads. (2) This Scheme requires shorter average transmission distance between $\mathrm{CHs}$ as well to the BS.

Some of the limitations in UCS as are: (1) Locating the CHs at the centre of the cluster is quite unrealistic as it is not suitable for most of the applications (2) Data communication between long distance node and BS would require more energy as inter-cluster transmission itself needs two hops.

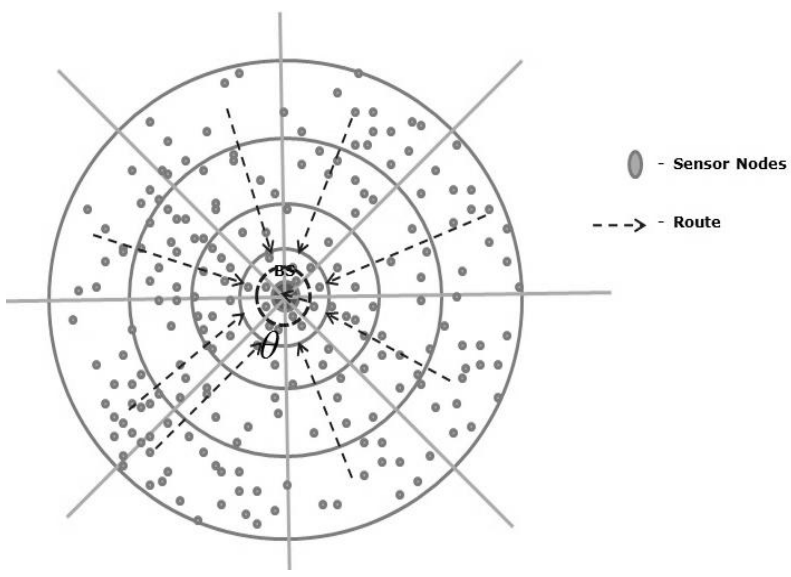

Figure 5. UCS Organization of Clusters

\section{COMPARATIVE STUDY ON CLUSTERING ROUTING PROTOCOLS}

In this section a comparative study on various clustering routing protocols that have been discussed in the previous section is being presented. Several metrics that are being impacted upon by routing such as scalability, energy efficiency, cluster stability, delay are analyzed in this comparison. The Table 1 enumerates the analytical framework for the clustering routing protocols 
Table 1. Comparison of various Clustering Routing Protocols in WSN

\begin{tabular}{|c|c|c|c|c|c|c|}
\hline Protocol & $\begin{array}{c}\text { Energy } \\
\text { Consumption }\end{array}$ & $\begin{array}{l}\text { Cluster } \\
\text { Stability }\end{array}$ & Scalability & Delivery Delay & Load Balancing & Robust \\
\hline LEACH & very low & moderate & very low & very small & moderate & Good \\
\hline HEED & moderate & high & moderate & moderate & moderate & low \\
\hline TL-LEACH & low & moderate & moderate & small & bad & low \\
\hline PEGASIS & low & low & very low & very large & moderate & Good \\
\hline TEEN & very high & high & low & small & good & Moderate \\
\hline APTEEN & moderate & very low & low & small & moderate & good \\
\hline MBC & moderate & low & Good & moderate & moderate & good \\
\hline BSCDCP & Very low & High & Very low & Small & good & Moderate \\
\hline TTDD & Very low & Very High & Low & Very Large & good & good \\
\hline UCS & Very Low & High & Good & small & good & Moderate \\
\hline
\end{tabular}

From the Table 1 statistics, it can be inferred that total energy consumption for all the clustered protocol are in the admissible range except TEEN. It is also noticed that cluster stability is intact when limited mobility is allowed. MBC and UCS scale well among other clustered protocols. Most protocols deliver data with minimum delay as they have a controlled mobility pattern. As the clusters have high stability for most of the protocols, the uniform load distribution is also realized.

\section{NEW RESEARCH DIRECTIONS}

Most of the routing protocols should have the general goal of sharing the route information from source to destination nodes in the network between the available routers.

However, in WSN scenario the major goals for developing routing protocols are improvement of network availability, increasing of the sensor network battery life time, efficient energy consumption[25], minimizing the delay in data transmission, and reducing of complexity of operation.

The WSNs have several limitations, such as limited energy supply, limited computing power, and limited bandwidth of the wireless links connecting sensor nodes. The main design goal of WSNs is to transmit data along with prolonging the lifetime of the network by employing efficient energy management strategies. The factors that influence WSN routing design are energy consumption, scalability and QoS.

Routing protocol design should be based on the application, architecture and size of the network.

In this work various hierarchical routing protocols have been compared, with which some issues related to clustering were identified. The energy hole problem in LEACH protocol could be addressed by periodically scanning the $\mathrm{CHs}$ for their energy levels. Uniformity of $\mathrm{CH}$ distribution can be achieved by employing grid techniques and moving the accumulated $\mathrm{CHs}$ to the grids that does not have a cluster head.

In HEED, forced CHs may not be used at all and may account for large energy loss for the entire network. These forced CHs should be identified and allowed them to act as member nodes along with being maintained in the $\mathrm{CH}$ reserve until its turn to become the next $\mathrm{CH}$.

As PEGASIS provides high delay in transmission, it is quite better to avoid using this scheme for large scale networks. PEGASIS can perform better in intra-cluster communications which require multi-hops to reach the $\mathrm{CH}$.

For TEEN and APTEEN schemes, employing multiple schemes would make them available to the nodes that are ready to transmit depending on the threshold value. In BSCDCP, the load at the BS would be quite high as it coordinates most of the actions of the nodes. Here as the BS could be mobile a periodic recharge of it is essential.

In TTDD, message transmission should be in a controlled fashion it doesn't become a colossal number of virtual grids.

UCS model of clustering offers energy efficient routing of data to the BS. Here energy efficiency can further be improved by rotating the cluster-heads in larger and highly populated clusters. Similarly one of the cluster-heads of smaller clusters that form next to BS can be assigned to forward data from other cluster-heads of that concentric circular region.

\section{CONCLUSION}

This paper provides a comprehensive study of the standard hierarchical routing protocol for wireless sensor networks. Here an attempt to identify the basic issues related to clustering type of routing protocols and their impact on energy consumption were done. A number of techniques in creating clusters were discussed, which is vital for data communication. The impact of mobility on clusters was analyzed and proper reconnection process was suggested. The difficulty of balancing the load over variable sized clusters was discussed. Cluster re-organizing is a way to uniformly distribute load among clusters. An elaborate comparative study on these clustered routing protocols provided good proof on the effectiveness of these protocols. We conclude that despite certain drawbacks, hierarchical or cluster type of routing in WSN surely consumes less energy and improves the overall life time of the wireless sensor network compared to other traditional routing protocols. In future we will be 
concentrating on the UCS model of clustering to devise a strategy to elect cluster-heads in larger clusters that can be in the range of other members as well able to forward their data to the next level.

\section{REFERENCES}

[1] Li C., Zhang H.X., Hao B.B., Li J.D., "A survey on routing protocols for large-scale wireless sensor networks", Sensors 2011, vol.11, pp.3498-3526.

[2] Al-Karaki, A. Kamal, "Routing Techniques in Wireless Sensor networks: A Survey," Security and Networks, 2004, Vol. 11, Issue 6, pp.6-28.

[3] Abbasi A.A., Younis M., "A survey on clustering algorithms for wireless sensor networks", Comput. Commun. 2007, vol.30, pp.2826-2841.

[4] Deosarkar B.P., Yada N.S., Yadav R.P., "Cluster Head Selection in Clustering Algorithms for Wireless Sensor Networks: A Survey", In Proceedings of the 2008 International Conference on Computing, Communication and Networking, Virgin Islands, USA, 3-7 August 2008; pp. 1-8.

[5] Xue Q, Ganz A., "Maximizing sensor network lifetime: analysis and design guides", In the Proceedings of the 2004 military communications conference, vol. 2, Monterey, CA, 2004. pp. 1144-1150.

[6] R.V. Biradar, V.C. Patil, S.R. Sawant, R.R. Mudholkar, "Classifiacation and Comparison of Routing Protocols in Wireless Sensor Networks," Special Issue on Ubiquitous Computing Security Systems, 2009, Vol. 4, Issue 2, pp. 704-711.

[7] Maimour M., Zeghilet H., Lepage F., "Cluster-based Routing Protocols for Energy-Efficiency in Wireless Sensor Networks" Available online: http://cdn.intechweb.org/pdfs/12423.pdf (accessed on 14 December 2010).

[8] G. Lukachan, M. Labrador, "SELAR: Scalable EnergyEfficient Location Aided Routing Protocol for Wireless Sensor Networks," In Proc. 29th Annual IEEE International Conference on Local Computer Networks, USA, Florida, 2004, pp. 694-695.

[9] R. Ogier, F. Templin, M. Lewis, "Topology Dissemination Based on Reverse-Path Forwarding (TBRPF)," RFC Editor 2004.

[10] Heinzelman W.R., Chandrakasan A., Balakrishnan H., "Energy-Efficient Communication Protocol for Wireless Microsensor Networks", In Proceedings of the 33rd Annual Hawaii International Conference on System Sciences, Maui, HI, USA, 4-7 January 2000, pp. 10-19.

[11] Tran-Quang V., Miyoshi T., "A Transmission Range Adjustment Algorithm to Avoid Energy Holes in Wireless Sensor Networks" In Proceedings of 8th AsiaPacific Symposium on Information and Telecommunication Technologies, Kuching, Malaysia, 15-18 June 2010, pp. 15-18.

[12] Younis O., Fahmy S., "HEED: A hybrid, energyefficient, distributed clustering approach for ad-hoc sensor networks", IEEE Transactions on Mobile Computing. 2004, vol.3, pp.366-379.

[13] S.C. Ergen, P. Varaiya, "On Multi-Hop Routing for Energy Efficiency," IEEE Commun. Lett., 2005, Vol. 9, Issue 10, pp. 880-881.
[14] Loscri V., Morabito G., Marano S., "A Two-Level Hierarchy for Low-Energy Adaptive Clustering Hierarchy", In Proceedings of the 2nd IEEE Semiannual Vehicular Technology Conference, Dallas, TX, USA, 25-28 September 2005; pp. 1809-1813.

[15] Liqi Shi, Fapojuwo A., "TDMA Scheduling with Optimized Energy Efficiency and Minimum Delay in Clustered Wireless Sensor Networks", IEEE Transactions on Mobile Computing, 2010, Vol.9, Iss.7, pp.927-940

[16] S. Lindsey, C. Raghavendra, "PEGASIS: PowerEfficient GAthering in Sensor Information Systems," In Proc. IEEE Aerospace Conference, USA, Montana, 2002, Vol. 3, pp. 1125-1130.

[17] Manjeshwar E., Agrawal D.P., "TEEN: A Routing Protocol for Enhanced Efficiency in Wireless Sensor Networks", In Proceedings of the 15th International Parallel and Distributed Processing Symposium (IPDPS), San Francisco, CA, USA, 23-27 April 2001 pp. 2009-2015.

[18] Lotf J.J., Hosseinzadeh M., Alguliev R.M., "Hierarchical Routing in Wireless Sensor Networks: A Survey" In Proceedings of 2010 2nd International Conference on Computer Engineering and Technology, Chengdu, China, 16-18 April 2010; pp. 650-654.

[19] Manjeshwar A., Agrawal D.P., "APTEEN: A Hybrid Protocol for Efficient Routing and Comprehensive Information Retrieval in Wireless Sensor Networks" In Proceedings of the 2nd International Workshop on Parallel and Distributed Computing Issues in Wireless Networks and Mobile computing, Lauderdale, FL, USA, 15-19 April 2002; pp. 195-202.

[20] Rong Ding, Bing Yang, Lei Yang, Jiawai Wang, "Soft Threshold based Cluster-Head Selection Algorithm for Wireless Sensor Networks", Third International Conference on Sensor Technologies and Applications, 2009, pp.526-530.

[21] S. Deng, J. Li, L. Shen, "Mobility based Clustering Protocol for Wireless Sensor Networks with Mobile Nodes", IET Wireless Sensor Systems, 2011, Vol.1, Iss.1, pp.39-47.

[22] S. Muruganathan, D. Ma, R. Bhasin, A. Fapojuwo, "A Centralized Energy-Efficient Routing Protocol for Wireless Sensor Networks," IEEE Commun. Mag., 2005, Vol. 43, Issue 3, pp. 8-13.

[23] H. Luo, F. Ye, J. Cheng, S. Lu, L. Zhang, "TTDD: TwoTier Data Dissemination in Large-Scale Wireless Sensor Networks," Wireless Networks, Springer Netherlands, 2005, Vol. 11, Issue 1, pp. 161-175.

[24] Soro S., Heinzelman W., "Prolonging the Lifetime of Wireless Sensor Networks via Unequal Clustering", In Proceedings of the 5th IEEE International Workshop on Algorithms for Wireless, Mobile, Ad Hoc and Sensor Networks (WMAN), Denver, CO, USA, 4-8 April 2005; pp. 236-243.

[25] Aslam N., Phillips W., Robertson W., Sivakumar S., "A multi-criterion optimization technique for energy efficient cluster formation in wireless sensor networks" Inf. Fusion 2011, vol.12, pp.202-212. 